\title{
Exercise effects on mood in breast cancer patients
}

\author{
H M van Oers, MA (Couns Psych) \\ Durban Oncology Centre, Durban, South Africa \\ Corresponding author: H M van Oers (fransvo@dtinc.co.za)
}

\begin{abstract}
Breast cancer is one of the most common cancers worldwide, and statistics reveal that the number of women diagnosed with breast cancer in South Africa is increasing. As such, medical practitioners will treat an increasing number of breast cancer patients. Although increasingly effective treatments improve patient survival intervals, a significant number of patients experience psychological distress, at the time of diagnosis and sometimes well beyond the start of treatment. This can be attributed to the disease itself and to treatment side-effects. Historically, patients experiencing such distress have been treated with pharmacotherapy or have been referred for psychotherapeutic intervention. Although it is well known that physical exercise is beneficial to physical health, only recently, and comparatively, has the effect of exercise been recognised as beneficial to psychological well-being. Cancer patients are often advised to reduce physical activity to avoid cancer-related fatigue. Paradoxically, recent research shows that physical exercise, of the type and intensity appropriate for the ability of each patient, can in fact play a significant role in improving mood and aiding physical recovery. This opens up a valuable additional resource to augment patients' quality of life, both physically and psychologically. One precaution stands vitally important, however: the prescribed exercise regimen must be tailored to the physical capabilities of the patient.
\end{abstract}

S Afr J SM 2013;25(2):55-59. DOI:10.7196/SAJSM.481

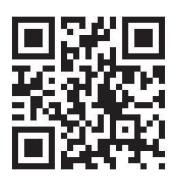

With over 1 million new cases diagnosed worldwide per annum, breast cancer continues to be the most common cancer affecting women. Unfortunately, surviving this cancer often means enduring significant and prolonged forms of adjuvant therapy, primarily radiation, chemotherapy and hormone therapy. These treatments, although increasingly effective in improving patient survival intervals, are toxic in numerous ways and produce negative short- and longterm physiological and psychological effects. ${ }^{[1]}$ Nearly all cancer survivors experience physiological and psychological symptoms and side-effects related to the disease or its treatment. ${ }^{[2]}$ Treatment-related symptoms, mood distress and decline in physical activity have all been found to be major problems encountered by cancer patients. ${ }^{[3]}$

While mortality decreases, the increase in cancer incidence means that the number of women receiving adjuvant cancer treatment, and living with the associated side-effects, is increasing. Therapyrelated negative side-effects include physiological and psychological symptoms such as pain, fatigue, muscle wasting, decreased cardiac function and psychological distress. Each symptom can contribute to a significant impairment of quality of life (QoL), and in some cases, affect prognosis. ${ }^{[3,4]}$ Depression is associated with chemotherapy non-compliance and reduced 5-year survival rates. ${ }^{[2]}$ Attention to psychological status is therefore an essential part of effective oncology treatment. However, in many instances, psychological distress is under-diagnosed and -treated due to time constraints, or because oncologists do not address or endorse this aspect of therapy.

Treatment effects on quality of life

Physiological sequelae

Differing treatment modalities can result in various physiological side-effects. Chemotherapy (e.g. anti-metabolites, anthracyclines and alkylators) is used to control suspected micrometastatic disease and typically consists of 6 - 8 cycles of polychemotherapy administered over approximately 21 weeks. Radiation is used following most breastconserving surgical procedures to control local disease and usually consists of daily treatment over a 6 -week period. Fatigue is a common side-effect of both of these treatment modalities. ${ }^{[5]}$ Chemotherapy has the further potential to cause decreased cardiorespiratory fitness and even cardiotoxicity in some patients, whereas radiation can also lead to irreversible lung fibrosis. Oral hormone therapy (progestins or anti-oestrogens administered daily over a 5 -year period) may cause ovarian ablation in pre-menopausal women, whereas the aromatase enzyme inhibitors prescribed for post-menopausal women may lead to fatigue and weight gain. Physical and functional deficits may also include asthenia, ataxia, muscle weakness and atrophy, hair-loss, sleep disturbances, nausea and vomiting, and pain. Many of these side-effects are common to patients with other cancers, but breast cancer patients face the additional burden of limited arm movement, lymphoedema of the arm, possible impaired cognitive function, weight gain and osteoporosis. ${ }^{[6]}$ In combination, these co-morbid effects can have a significant negative effect on QoL..$^{[3,7]}$

\section{Psychological sequelae}

Recent studies have estimated the prevalence of depression, including major and minor depressive episodes and dysthymia, to be in the order of $20 \%$, and the prevalence of anxiety disorders to be approximately $10 \% \cdot{ }^{[8]}$ Depression is characterised by feelings of sadness, hopelessness, changes in sleep and eating habits, psychomotor retardation and withdrawal from social contacts, which in turn lead to a deterioration of QoL, and impaired social and occupational functioning. ${ }^{[7]}$ Cancer patients may show scores twice as high as those of the general population on the anxiety and depression subscales 
of the Hospital Anxiety and Depression Scale. ${ }^{[4]}$ Other psychological and emotional side-effects experienced by cancer patients include stress, fear of death, poor body image, low self-esteem, loneliness and a sense of loss of control over life. ${ }^{[2,3]}$ One study ${ }^{[0]}$ found a similar deterioration in fatigue and QoL measures in breast cancer patients shortly after completing adjuvant therapies. QoL impairment was also strongly associated with scores on depression, as well as fatigue. ${ }^{[9]}$

Despite an inconclusive relationship between psychological distress and cancer survival, a review of available research relating to breast cancer shows that most studies indicate a significant relationship between psychological factors and survival. ${ }^{[10,11]}$ Clinical depression is associated with non-compliance with cancer treatment and reduced survival, emphasising that depression negatively affects the psychological and physical health of many patients. ${ }^{[2,7]}$ This underlines the role of psychological factors in cancer survival and emphasises the need for optimal supportive care to facilitate positive effects on such prognostic factors. It is clear that the appropriate management of psychological distress is of clinical importance, and it is vital that oncology healthcare professionals are aware of the importance of patients' psychological functioning.

Current treatments for anxiety and depression in cancer patients include pharmacotherapy and psychotherapeutic interventions. For many, these treatments are safe, effective and provide significant improvements; however, there are some patients in whom these existing interventions are less effective due to personal, behavioural or organic factors. For example, selective serotonin-reuptake inhibitors (SSRIs) may be contraindicated in patients receiving hormone blockade therapies. In the general population, exercise acts as an effective antidepressant; it may therefore serve as an alternative form of treatment to provide symptom relief for depression and anxiety and improve physical health outcomes in cancer patients. ${ }^{[7,10]}$

\section{The effects of exercise on mood}

It is well known that physical exercise is beneficial to the body, but it is only recently, and comparatively, that the effect of exercise has been studied in terms of its effect on mood. Mental and physical health are closely associated and can exert a significant effect on one another: mental health can be affected positively by physical exercise, and conversely, patients involved in physical rehabilitation tend to exhibit better compliance with, and make greater advances in treatment when they feel positive. ${ }^{[12]}$

Exercise increases blood flow and circulation, can improve memory function and provides a feeling of general well-being. There is growing evidence that physical activity and exercise have positive effects on mood in general, and on depression and anxiety in particular. ${ }^{[7,13-17]}$ The manner in which exercise improves psychological well-being is explained by a neurobiological response and how physical exertion relates to the fight-or-flight response.

Most research has focused on hormonal reaction to increased physical activity, in particular the increased levels of beta-endorphins, encephalins, catecholamines and serotonin. Endorphins are released by the pituitary gland during sustained vigorous exercise. These serve to reduce stress, decrease appetite and improve immune function. Serotonin, together with norepinephrine, is a neurotransmitter that regulates mood and can affect anxiety mechanisms, thoughts and sleep patterns. Studies have shown that low concentrations of serotonin cause symptoms of depression. The mechanisms involved in the increase of serotonin levels after exercise may be the result of motor activity increasing the firing rates of serotonin neurons, causing an increase in its release and synthesis. By increasing in concentration, these neurotransmitters serve to improve mood and decrease symptoms of depression. ${ }^{[2,18]}$

In the general population, exercise is an effective antidepressant; individuals with moderate to more severe depression benefit comparably, and exercise is equally effective for men and women across a wide range of ages. These effects have been found to be comparable with psychotherapy and medication, particularly for those with mild to moderate depression. ${ }^{[7]}$ The antidepressant effects of exercise have also been shown to last long beyond the exercise period. ${ }^{[13]}$ Yet, while the majority of studies concur regarding the positive effects of physical activity on mood, there is still uncertainty regarding which form of exercise is best for improving mood and mental health, and the specific effects of different types of exercise. ${ }^{[19]}$

\section{Effects of different forms of exercise}

The main forms of exercise are aerobic and anaerobic. Aerobic exercise occurs when oxygen is metabolised to produce energy. This is generally attained through sustained periods of hard work and vigorous activity lasting longer than 3 minutes at a time. Anaerobic exercise refers to physical exertion where the energy required to produce this activity is provided independently of oxygen. This type of exercise uses short bursts of vigorous activity and typically lasts less than a minute. There are also alternative forms of activity that do not readily fall into the definitions of aerobic or anaerobic, but which also have the potential to affect mood and emotional well-being (e.g. yoga and meditation). ${ }^{[12]}$

Research suggests that aerobic exercise has the greatest moodelevating effects when performed continuously over an extended period of time, where the release of endorphins is responsible for the feel-good effect or 'runner's high' that athletes experience after bouts of vigorous activity ${ }^{[12]}$ In addition to this endorphin-related positive effect, cortisol levels are altered by exercise: 30 minutes of moderate aerobic activity can reduce cortisol levels, higher levels of which are associated with negative affective states. This reduction may partly explain the moodelevating effects of physical activity. A factor influencing the degree to which aerobic exercise is beneficial appears to be the intensity: low- to moderate-intensity exercise has been shown to be effective in reducing both anxiety and depression, ${ }^{[20]}$ whereas high-intensity exercise has been found in some instances to induce the opposite effect and increase levels of anxiety. ${ }^{[21]}$ Furthermore, moderate-intensity exercise may produce more sustained psychological benefits, probably because it is easier to maintain over longer periods of time. ${ }^{[12]}$

It is generally believed that anaerobic exercise provides similar mood-enhancing effects to aerobic exercise only when performed at high-intensity levels. This is possibly due to the build-up of lactate in the muscles. While most studies show no difference between these two forms of exercise in terms of their mood-elevating potential, some studies have shown that anaerobic exercise does not confer the moodelevating effects attributed to aerobic exercise. ${ }^{[22]}$ Higher-intensity anaerobic exercises are more likely to lead to injuries, such as muscle tears and strains; as such, this aspect may outweigh its benefits with respect to positive emotional effects. ${ }^{[12]}$ 
Additionally, low-intensity aerobic exercises such as yoga and tai chi have been found to have positive effects on psychological functioning, leading to decreases in anxiety. Tai chi, as a slower and less intense aerobic exercise, has been found to be a suitable alternative for patients not capable of more intensive exercise programmes. ${ }^{[12]}$

\section{Exercise and the cancer patient}

Until recently, patients were often advised to reduce their levels of physical activity and to seek more frequent periods of rest in order to counteract cancer-related fatigue. Paradoxically, such a decrease in physical activity can, in fact, compound symptoms of fatigue, since a more sedentary lifestyle induces muscle catabolism and may cause further deficits in functional capacity. There is growing scientific evidence that exercise can substantially reduce cancer-related fatigue, which is linked to psychological distress, and improve QoL. ${ }^{[23,24]}$

Several studies have shown that exercise is likely to mediate fatigue, anxiety, depression and QoL in cancer patients. ${ }^{[3,7,25-27]}$ One study found that exercise leads to significant decreases in fatigue, anxiety and depression in breast cancer patients receiving adjuvant therapy, with greater decreases with low weekly exercise doses. The study stated that a 20-week exercise programme comprised of a session of resistance training for principal muscle groups and 2 moderate-intensity aerobic sessions per week, each lasting 30 - 45 minutes, would be optimal in effecting positive mood change in these patients. This was found to be preferable to a higher-dose intervention. ${ }^{[4]}$ Another study found that light- to moderate-intensity aerobic exercise decreased anxiety in a sample of breast cancer patients, and indicated that this may be a valuable anxiolytic tool. ${ }^{[28]}$

Other research showed that exercise produced modest effects on depression in breast cancer patients across various disease stages. ${ }^{[7]}$ Nine studies of breast cancer patients were reviewed; all included programmes of aerobic and strength-training components initiated either prior to, or during adjuvant treatment. The review did not target depression through the selection of depressed cancer patients or by selecting exercises known to have the greatest effects on depression in other populations; therefore, in reality, the positive effects may be even greater for patients actually experiencing significant depression and targeted with exercise interventions effecting optimal change. These findings indicated that exercise produced the largest antidepressant effects when carried out over sessions longer than 30 minutes, and in terms of frequency, exercising 5 times per week was significantly more effective than 2 - 4 days weekly. In terms of intensity, it was found that light- to moderate-intensity exercise proved to be an effective antidepressant; with respect to breast cancer patients, light-intensity programmes are preferable as they are easier to incorporate into patient routines, and therefore result in greater compliance and exercise frequency.

Stagl ${ }^{[26]}$ found that women who exercised the most between surgery and starting treatment were the least affected by depression and fatigue. More physically active patients tended to exhibit greater confidence in their ability to continue with their routine domestic, occupational and social activities. This, in turn, brought about greater feelings of satisfaction and led to appraisals of less depression and higher QoL. The recommended levels of exercise were 20 minutes daily for the greatest benefit, but exercising every other day also proved to be therapeutic. The study emphasised that exercise can take many forms, e.g. jogging, cycling and swimming. ${ }^{[26]}$
Whereas most studies focus on exercise as a planned activity with the goal of achieving fitness, one study found that other forms of physical exertion, including occupational and household activities, were also effective in alleviating symptoms of psychological distress. ${ }^{[23]}$ Patients involved in any form of physical activity were less likely to report anxiety and depression in a preceding 30 -day period, than those who were totally sedentary. The study found moderate-intensity activity to be optimal for breast cancer patients, with multiple short bouts of activity resulting in the greatest improvements. ${ }^{[23]}$

Regarding the aforementioned studies, the timing of exercise interventions and whether patients received adjuvant treatment in the post-diagnostic period need to be considered. Some studies examined exercise effects in patients receiving adjuvant treatment, while the patients in other studies had completed active treatment. Some research found that treatment status at baseline was not significant. This suggests that exercise may lessen symptoms of depression among patients who are undergoing treatment during exercise interventions, compared with those who are not. For many patients, depression may resolve after diagnosis and treatment, but for many it may persist or even develop after the treatment period is over. ${ }^{[7]}$

While there is general consensus regarding the efficacy of exercise as a mediator of psychological distress in breast cancer patients, the majority of studies acknowledge that any form of recommended activity of necessity must consider the limitations placed on each patient in terms of their physical status in response to their disease and specific oncological treatment. Recommendations should be tailored for each patient with respect to the intervention period, i.e. during v. postadjuvant therapy, in terms of the exercise type and intensity that the patient is able to tolerate. One study states that the specific beneficial effects of exercise, both during and after treatment, may vary as a function of the stage of disease, the nature of the medical treatment and the current lifestyle of the patient. ${ }^{[29]}$

Patients receiving adjuvant therapy experience patterns of fatigue that increase with chemotherapy cycles and peak 5 days after therapy administration. Exercise programmes for such patients need to be adapted to progressively low to moderate doses of exercise across chemotherapy cycles, with fewer exercise sessions in the 10-day period following infusion, increasing to more frequently in the days thereafter. It has further been found that low doses of exercise (90 - 120 minutes of moderate exercise weekly) are more effective than higher doses. ${ }^{[4]}$

Breast cancer patients can adhere to a conventional exercise programme, even during adjuvant treatment. There is evidence to suggest that walking is the natural choice for most patients, and that cycle ergonometry offers the advantages of a sitting position and leg exercises (minimising ataxia, limitations in upper-body movement and lymphoedema). Upper-body exercises are not contraindicated, but patients should be advised that wearing a compression sleeve on the arm of the affected side is a wise precaution. ${ }^{[27]}$

Cancer patients who are able to, should exercise aerobically (e.g. brisk walking or static cycling) for 20 - 60 minutes 3 - 5 days weekly. More debilitated patients should be advised to engage in several shorter exercise sessions per day, and that the exercise programme can progress as the patient becomes fitter. Most importantly, the physician should always be aware that every patient will adapt differently to the exercise stimulus and goals; physical tolerance and age should determine individual rate of progress. ${ }^{[1]}$ 
Some studies have found that the location and supervision of the exercise intervention are important; the greatest optimal effects have been observed in patients who participate in programmes that are supervised and exercise in facilities. Home-based and unsupervised exercise interventions have, in fact, been associated with an increase in depressive symptoms. ${ }^{[2,7]}$

\section{Contraindications to exercise}

Medical screening is an essential aspect of prescribing an exercise programme for any cancer patient. Several medical factors constitute general contraindications to exercise: cardiovascular insufficiency, such as acute myocarditis or recent myocardial infarction; acute infectious diseases; metabolic diseases such as thyrotoxicosis; and any physical impairment leading to inability to exercise. ${ }^{[1]}$

In addition to these general contraindications, certain precautions are specific to cancer patients. Exercise should be avoided 24 hours prior to intravenous chemotherapy. Exercise should also be avoided at least 2 hours after either chemotherapy infusion or radiation therapy, as increased circulation may affect treatment. Anaemic patients should avoid exercise until the anaemia has improved. Onset of nausea during exercise and vomiting within 24 - 36 hours of exercise, feelings of unusual fatigue or muscle weakness, blurred vision, faintness or any pain unassociated with injury are also regarded as contraindications to continued exercise.

In addition, immune-compromised patients should avoid public gyms until their white blood cell count returns to safer levels. ${ }^{[1]}$

\section{Further research}

There is some disparity in the literature about the effectiveness of exercise in terms of different sub-populations. For example, age, socioeconomic status, ethnicity and employment status are not included or accounted for in the majority of studies. A recent study found that women of low socio-economic status with breast cancer have an increased risk of developing depression, and that the symptom burden may differ by socio-economic status and ethnicity. For example, older African-American patients who were unemployed were 3 times more likely to become depressed than those who were employed. Another study found differences in levels of activity and QoL between white breast cancer patients and patients from minority groups. Physical activity was associated with increased QoL in white patients, but not in minority group women. ${ }^{[30]}$ More research is needed to better identify and treat breast cancer patients at risk for psychological distress.

While there has been some research on the use of exercise as an intervention for breast cancer patients in South Africa (SA), much of this literature is focused on the physiological improvements that an exercise intervention can confer within the context of physiotherapy and rehabilitation, ${ }^{[31]}$ or the positive effects on brain function. ${ }^{[32]}$ There seems to be a dearth of research into the efficacy of such interventions as a therapeutic option for psychological distress on the SA population; much less so on the oncology patient population, especially given the diversity of composite ethnicities, cultures and socio-economic groups. As stated above, several studies have noted that socio-economic and employment status as well as cultural factors were found to be considerations in terms of risk of depression and symptom burden. Many SA patients may benefit considerably from an exercise intervention that may be more cost-effective and attainable than medication and has the benefit of providing a means of improving their health on multiple levels.

\section{Conclusion}

It has been known for some time that physical activity exerts a positive effect on mood in the general population, and with later research, that it holds promise for improving symptoms of psychological distress in oncology patients. What is becoming increasingly clear is that exercise may be a valuable addition to the therapeutic resources of physicians and oncology healthcare professionals. Where depression and anxiety in cancer patients have historically been treated using pharmacotherapy or psychotherapy, recommending an exercise programme may contribute significantly to physical and psychological well-being in a non-intrusive, non-toxic and accessible manner. Importantly though, certain caveats remain: physical status must be regarded as paramount in terms of the type and quantity of exercise that the breast cancer patient is capable of performing, given the limitations imposed by surgery, lymphoedema, treatmentrelated fatigue and pre-diagnostic levels of fitness. Further research is needed to identify appropriate exercise regimens for different subpopulations of breast cancer patients to optimise the benefits of such an intervention.

\section{References}

1. Rajarajeswaran P, Vishnupriya R. Exercise in cancer. Ind J Med Paed Oncol 2009; 30(2):6170. [http://dx.doi.org/10.4103\%2F0971-5851.60050]

2. Brown JC, Huedo-Medina TB, et al. The efficacy of exercise in reducing depressive symptoms among cancer survivors: A meta-analysis. PLoS ONE 2012;7(1). [http://dx.doi. org/10.1371/journal.pone.0030955]

3. Yang CY, Tsai JC, Huang YC, Lin CC. Effect of a home-based walking program on perceived symptom and mood status in postoperative breast cancer women receiving adjuvant chemotherapy. J Adv Nurs 2011;67(1):158-168: [http://dx.doi.org/10.1111 /j.1365-2468.2010.05492]

4. Carayol M, Bernard P, Boiche J, et al. Psychological effect of exercise in women with breast cancer receiving adjuvant treatment therapy: What is the optimal dose needed? Ann Oncol 2013;24(2): 291-300. [http://dx.doi.org/10.1093\%2Fannonc\%2Fmds342]

5. Schmidt ME, Chang-Claude J, Vrieling A, Heinz J, Flesch-Janys D, Steindorf K. Fatigue and quality of life in breast cancer survivors: Temporal courses and long-term pattern. J Cancer Surviv 2012;6(1):11-19. [http://dx.doi.org/10.1007\%2Fs11764-011-0197-3]

6. Pinto AC, de Azambuja E. Improving quality of life after breast cancer: Dealing with symptoms. Maturitas 2011;70(4):343-348. [http://dx.doi.org/10.1016/j. maturitas.2011.09.008]

7. Craft LL, Vaniterson EH, Helenowski IB, et al. Exercise effects on depressive symptoms in cancer survivors: A systematic review and meta-analysis. Cancer Epidemiol Biomarkers Prev 2012;20(1):3-19. [http://dx.doi.org/10.1158\%2F1055-9965.EPI-110634]

8. Mitchell AJ, Chan M, Bhatti H, et al. Prevalence of depression, anxiety and adjustment disorder in oncological, haematological and palliative care settings: A meta-analysis of 94 interview-based studies. Lancet Oncol 2011;12(2):160-174. [http://dx.doi. org/10.1016\%2FS1470-2045\%2811\%2970002-X]

9. Penttinen HM, Saarto T, Kellokumpu-Lehtinen P, et al. Quality of life and physical performance and activity of breast cancer patients after adjuvant treatments. Psychooncology 2011;20(11):1211-1220. [http://dx.doi.org/10.1002/pon.1837]

10. Chen X, Lu W, Zheng Y, et al. Exercise, tea consumption and depression among breast cancer survivors. J Clin Oncol 2010;28(6):991-998. [http://dx.doi. org/10.1200\%2FJCO.2009.23.0565]

11. Falagas ME, Zarkadoulla EA, Ioannidou EN, et al. The effect of psychosocial factors on breast cancer outcome: A systematic review. Breast Cancer Res 2007;9(4):R44. [http:// dx.doi.org/10.1186\%2Fbcr1744]

12. Cohen G, Shamus E. Depressed, low self-esteem: What can exercise do for you? Internet J Allied Health Sci Pract 2009;7:2.

13. Scully D, Kremer J, Meade MM, Graham R, Dudgeon K. Physical exercise and psychological well being: A critical review. Br J Sports Med 1998;32:111-120. [http:// dx.doi.org/10.1136/bjsm.32.2.111] 
14. Guszkowska M. Effects of exercise on anxiety, depression and mood. Psychiatr Pol 2004;38(4):611-620

15. Strohle A. Physical activity, exercise, depression and anxiety disorders. J Neural Transm 2009;116(6):777-784. [http://dx.doi.org/10.1007\%2Fs00702-008-0092-x]

16. Hallgren MA, Moss ND, Gastin P. Regular exercise participation mediates the affective response to acute bouts of vigorous exercise. J Sports Sci Med 2010;9:629-637.

17. McHugh J, Lawlor BA. Exercise and social support are associated with psychological distress outcomes in a population of community-dwelling older adults. J Health Psychol 2012;17(6):833-844. [http://dx.doi.org/10.1177\%2F1359105311423861]

18. Young SN. How to increase serotonin in the human brain without drugs. J Psychiatr Neurosci 2007;32(6):394-399.

19. Shamus E, Russo SA, Fields C, Peal G, Marikle SQ, Butler RD. Exercise and mental health: Psychological benefits. Osteo Fam Phys News 2008;8(5):1-8.

20. Rendi M, Szabo A, Szabo T, Velenczei A, Kovacs A. Acute psychological benefits of aerobic exercise: A field study into the effects of exercise characteristics. Psychol Health Med 2008;13(2):180-184

21. Bixby WR, Hatfield BD. A dimensional investigation of the State Anxiety Inventory (SAI) in an exercise setting: Cognitive vs. somatic. J Sport Behav 2011;34(4):307-324

22. Callaghan P. Exercise: A neglected intervention in mental health care? J Psychiatr Ment Health Nurs 2004;11:476-483. [http://dx.doi.org/10.1111\%2Fj.1365-2850.2004.00751.x]

23. Basen-Engquist K, Hughes D, Perkins H, Shinn E, Carmack Taylor C. Dimensions of physical activity and their relationship to physical and emotional symptoms in breast cancer survivors. J Cancer Surviv 2008;2(4):253-261. [http://dx.doi.org/10.1007\%2 Fs11764-008-0067-9]
24. Arnold M, Taylor NF. Does exercise reduce cancer-related fatigue in hospitalised oncology patients? A systematic review. Onkologie 2010;33(11):625-630. [http://dx.doi. org/10.1159\%2F000321145]

25. Loprinzi PD, Cardinal BJ. Effects of physical activity on common side effects of breast cancer treatment. Breast Cancer 2012;19(1):4-10.

26. Stagl JM. Benefits of physical activity on depression and functional quality of life during treatment for breast cancer: Psychosocial mechanisms. Open Access Theses, 2011: Paper 278.

27. Courneya KS, Mackey JR, McKenzie DC. Exercise for breast cancer survivors: Research evidence and clinical guidelines. Phys Sportsmed 2002;30(8):33-42.

28. Blacklock R, Rhodes R, Blanchard C, Gaul C. Effects of exercise intensity and self-efficiency on state anxiety with breast cancer survivors. Oncol Nurs Forum 2010;37(2):206-212. [http://dx.doi.org/10.1188\%2F10.ONF.206-212]

29. Knols R, Aaronson NK, Uebelhart D, Fransen J, Aufdemkampe G. Physical exercise in cancer patients during and after medical treatment: A systematic review of randomised and controlled clinical trials. J Clin Oncol 2005;23:3830-3842. [http://dx.doi. org/10.1200\%2FJCO.2005.02.148]

30. Mandelblatt JS, Luta G, Kwan M, et al. Associations of physical activity with quality of life and functional ability in breast cancer patients during active adjuvant treatment: The Pathways Study. Breast Cancer Res Treat 2011;129(2):521-529. [http://dx.doi.org/10.100 7\%2Fs10549-011-1483-5]

31. Derman EW, Whitesman S, Dreyer M, et al. Healthy lifestyle interventions in general practice: Part 5: Lifestyle and cancer. SA Fam Pract 2009;51(2):91-95.

32. Stein DJ, Collins M, Daniels W, Noakes TD, Zigmond M. Mind and muscle: The cognitive-affective neuroscience of exercise. CNS Spectr 2007;12(1):19-22. 